\title{
Physical fitness affects the quality of single operator cardiocerebral resuscitation in healthcare professionals
}

\author{
Dominique Hansen $^{\mathrm{a}, \mathrm{b}, \mathrm{d}}$, Pascal Vranckx ${ }^{\mathrm{c}, \mathrm{e}}$, Tom Broekmans ${ }^{\mathrm{b}, \mathrm{d}}$, \\ Bert O. Eijnde ${ }^{\mathrm{b}, \mathrm{d}}$, Walter Beckers ${ }^{\mathrm{a}}$, Philippe Vandekerckhove ${ }^{\mathrm{e}}$, \\ Paul Broos ${ }^{\mathrm{e}, \mathrm{f}}$ and Paul Dendale ${ }^{\mathrm{a}, \mathrm{b}, \mathrm{d}}$
}

\begin{abstract}
Objective Sustained external chest compressions during cardiocerebral resuscitation (CCR) are physically demanding. It might be hypothesized that a high cardiopulmonary exercise capacity and/or muscle strength delays the development of physical fatigue and, consequently, preserves CCR quality. We intended to assess the impact of cardiopulmonary exercise capacity and muscle strength on CCR quality.
\end{abstract}

Methods Fifteen healthcare professionals (10 men and five women, mean age $34 \pm 9$ years) performed a 15-min hands-on CCR session on an adult training manikin. CCR compression depth (from which CCR quality was calculated) and frequency were monitored. During CCR we assessed serial blood lactate concentrations, and provided continuous heart rate monitoring. Relationships were examined between participant characteristics, peak cardiopulmonary exercise capacity, ventilatory threshold, maximal muscle strength, muscle strength endurance and CCR quality.

Results Significant univariate correlations were found between 15-min CCR quality and body height $(r=0.53)$, ventilatory threshold $(r=0.67)$, peak oxygen uptake capacity $(r=0.54)$, peak cycling power output $(r=0.54)$, and maximal isometric elbow extension strength $(r=0.55)$ $(P<0.05)$. CCR quality was significantly lower in females, when compared with males $(P<0.05)$. Within different timeframes, CCR quality was mainly related to the

\section{Introduction}

Following cardiac arrest, maintenance of cerebral and coronary perfusion is critical to neurological outcome and patient survival [1]. Cardiocerebral resuscitation (CCR) without mouth-to-mouth ventilations, and prompt cardiac defibrillation, provides a simple and effective approach for witnessed cardiac arrest [2].

It is well established that the critical issues for good perfusion pressures and blood flow during bystander resuscitation efforts are the force and rate of compressions, full recoil of chest wall after each compression and minimized interruptions. In studies of CCR, applied

The opinions expressed in this article are those of the authors and are not necessarily those of Red Cross Flanders. ventilatory threshold up to the first $5 \mathrm{~min}(P<0.05)$, whereas CCR quality was mainly related to maximal isometric elbow extension strength after $5 \mathrm{~min}(P<0.05)$.

Conclusion In healthcare professionals, the ventilatory threshold is significantly related to CCR quality during the first few min. Healthcare professionals who are regularly involved in CCR should therefore aim to achieve/sustain a high aerobic exercise capacity.

Clinical trial registration information Study registration number: ISRCTN70447230, www.controlled-trials.com/ ISRCTN70447230. European Journal of Emergency Medicine 00:000-000 @ 2011 Wolters Kluwer Health | Lippincott Williams \& Wilkins.

European Journal of Emergency Medicine 2011, 00:000-000

Keywords: exercise tolerance, quality, resuscitation

${ }^{a}$ Heart Centre Hasselt/Jessa Hospital, ${ }^{b}$ Rehabilitation Research Centre, Department of Healthcare, PHL-University College, ' Department of Cardiac Intensive Care, Heart Centre Hasselt, d'Hasselt University, Faculty of Medicine, Diepenbeek, ${ }^{\mathrm{e}}$ Red Cross Flanders and ${ }^{\mathrm{f}}$ Department of Surgery, University Hospital Gasthuisberg, Leuven, Belgium

Correspondence to Pascal Vranckx, MD, Heart Centre Hasselt/Jessa Hospital, Stadsomvaart 11, 3500 Hasselt, Belgium

Tel: +32 11309589; fax: +32 11309328; e-mail: pascal.vranckx@jessazh.be

Hansen Dominique and Pascal Vranckx equally contributed to the writing of the article

Received 19 November 2010 Accepted 8 April 2011

by healthcare professionals, in out-of-hospital [3] and in-hospital settings [4], approximately $40 \%$ of chest compressions were of insufficient depth. It might be hypothesized that rescuer physical fatigue might interfere with delivery of adequate chest compression (i.e. rate or depth). Significant physical fatigue and shallow compressions are seen after already $1 \mathrm{~min}$ of CCR, although rescuers may deny that physical fatigue is present within $5 \mathrm{~min}$ [5].

Physical fatigue can be defined as 'the decreased capacity or incomplete ability of an organism, an organ, or a part to function normally because of excessive stimulation or prolonged exertion' [6]. The workload at which physical fatigue occurs is, at least in part, determined by the physical fitness. The physical fitness is composed of two main features: cardiopulmonary (or physical) exercise 
capacity and muscle strength. As a result, it may be speculated that a reduced cardiopulmonary exercise capacity and/or muscle strength interferes with proper CCR performance. However, it remains to be established whether there is a correlation between these components of the general physical fitness and CCR performance [7].

The aim of this study is to evaluate the impact of cardiopulmonary exercise capacity and muscle strength on the performance of 15 -min sustained, continuous external chest compressions of only CCR of healthy healthcare professionals.

\section{Methods \\ Participants}

Fifteen (10 men, see Table 1) active healthcare professionals (12 intensive cardiac care nurses, three physicians), between the age of 21 and 52 years, were included in this study. These participants were selected as they work at hospital departments where CCR is regularly executed (cardiac intensive care and emergency care units), and receive a yearly refamiliarisation course. All the participants were required to be able to achieve a maximal voluntary cardiopulmonary exercise test. All the participants were informed in detail about the nature and risks of this study and provided written informed consent. The study protocol was approved by the local Institute Review Board according to the Helsinki declaration. The participants were excluded from participation in the study in case of any chronic disease and/or orthopaedic injury/dysfunction.

\section{Study design, methodology and definitions}

Following participant inclusion, the participants performed a cardiopulmonary exercise test and muscle strength test ('physical fitness tests') on two separate days interspersed by at least 48 -h recovery. Hereafter, the participants received standardised CCR familiarization ('instruction phase'). Fourteen days after CCR familiarisation, quality of CCR, heart rate and blood lactate concentrations were assessed during a 15-min sustained CCR session ('evaluation phase', see Fig. 1). All measurements were performed by the same investigator at the same time of the day. During the study period, the participants have not executed real CCR.

\section{Physical fitness tests Cardiopulmonary exercise capacity}

All the participants performed a maximal incremental 1-min stage cardiopulmonary cycloergometer exercise test [10] as executed in previous studies from our laboratory [11]. Following criteria were used to define maximal exercise effort during exercise testing: respiratory exchange ratio of greater than $1.10 \mathrm{and} /$ or heart rate greater than $95 \%$ of maximal predicted value. During the cycloergometer test, an electronically braked Ergo 1500 cycle (ErgoFit, Pirmasens, Germany) was used. The cycling frequency was set at $70 \mathrm{rpm}$ and the test was ended when the participant failed to maintain a cycling frequency of at least $60 \mathrm{rpm} \mathrm{[12].} \mathrm{Before} \mathrm{every} \mathrm{test} \mathrm{an}$ automatic gas and volume calibration was performed and during the tests environmental temperature was kept stable at $20^{\circ} \mathrm{C}$. During the exercise tests pulmonary gas exchange analysis was performed by a cardiopulmonary ergospirometry device (Schiller CS200, Schiller AG, Switzerland). Oxygen uptake capacity and carbon dioxide output were collected breath-by-breath and averaged every $10 \mathrm{~s}$. Peak oxygen uptake capacity $\left(\mathrm{VO}_{2 \text { peak }}\right)$ is the maximum capacity of an individual's body to transport and use oxygen during incremental exercise, which reflects the physical fitness of the individual. Ventilatory threshold

Table 1 Baseline participant characteristics, maximal exercise performance capacity and muscle strength: univariate correlations with cardiocerebral resuscitation quality

\begin{tabular}{|c|c|c|c|c|c|c|c|}
\hline & \multirow[b]{2}{*}{ Mean $\pm S D$} & \multirow[b]{2}{*}{$\begin{array}{l}\text { Correlation with overall } \\
\text { CCR quality }(r)\end{array}$} & \multicolumn{5}{|c|}{$\begin{array}{c}\text { Correlation with CCR quality within different } \\
\text { timeframes }(r)\end{array}$} \\
\hline & & & $<1 \min$ & $1-3 \min$ & $3-5 \min$ & $5-10 \min$ & $10-15 \mathrm{~min}$ \\
\hline Age (years) & $34.5 \pm 9.4$ & 0.20 & 0.28 & 0.39 & 0.51 & 0.10 & 0.10 \\
\hline BMI $\left(\mathrm{kg} / \mathrm{m}^{2}\right)$ & $24.1 \pm 2.6$ & 0.10 & 0.10 & 0.17 & 0.10 & 0.10 & 0.10 \\
\hline Height $(\mathrm{cm})$ & $174 \pm 8$ & $0.53^{*}$ & 0.45 & 0.51 & $0.54 *$ & 0.37 & 0.37 \\
\hline Weight $(\mathrm{kg})$ & $73 \pm 11$ & 0.43 & 0.20 & 0.10 & 0.26 & 0.26 & 0.14 \\
\hline $\mathrm{VO}_{2 \text { peak }}(\mathrm{ml} / \mathrm{min})$ & $2884 \pm 755$ & $0.54^{*}$ & 0.45 & $0.53 *$ & 0.39 & 0.40 & 0.47 \\
\hline$\%$ predicted $\mathrm{VO}_{2 \text { peak }}$ & $117 \pm 31$ & 0.10 & 0.20 & 0.20 & 0.10 & 0.10 & 0.10 \\
\hline $\mathrm{W}_{\max }$ & $253 \pm 75$ & $0.54^{*}$ & 0.41 & $0.52 *$ & 0.42 & 0.42 & 0.48 \\
\hline Ventilatory threshold $(\mathrm{ml} / \mathrm{min})$ & $2061 \pm 593$ & $0.67^{*}$ & $0.62 *$ & $0.73 *$ & $0.71^{*}$ & 0.45 & 0.14 \\
\hline Ventilatory threshold/ $/ \mathrm{VO}_{2 \text { peak }}$ ratio $(\%)$ & $70 \pm 7$ & 0.10 & 0.10 & 0.10 & 0.20 & 0.17 & 0.14 \\
\hline Isometric elbow extension peak torque at $90^{\circ}(\mathrm{Nm})$ & $55 \pm 19$ & $0.55^{*}$ & 0.24 & 0.40 & 0.42 & $0.60 *$ & $0.63^{*}$ \\
\hline Isokinetic elbow extension work fatigue at $270 \%$ s (\%) & $20 \pm 9$ & 0.20 & 0.10 & 0.14 & 0.22 & 0.42 & 0.51 \\
\hline Maximal hand grip strength $(\mathrm{kg})$ & $49 \pm 11$ & 0.45 & 0.39 & 0.50 & 0.50 & 0.42 & 0.51 \\
\hline$\%$ Predicted maximal hand grip strength & $130 \pm 22$ & 0.20 & 0.10 & 0.10 & 0.10 & 0.24 & 0.14 \\
\hline
\end{tabular}

Spearman's rank correlation coefficients are represented $(n=15)$.

Isometric elbow extension peak torque at $90^{\circ}$ indicates maximal muscle strength. Isokinetic elbow extension work fatigue at $270 \%$ (s $\%$ ) indicates muscle strength endurance. Predicted maximal hand grip strength based on Schlüssel et al. [8]. Predicted maximal $\mathrm{VO}_{2 \text { peak }}$ based on Wasserman et al. [9].

$\mathrm{SD}$, standard deviation; $\mathrm{VO}_{2 \text { peak }}$, peak oxygen uptake capacity; $\mathrm{W}_{\max }$, maximal cycling power output.

*Significant correlation $(P<0.05)$. 


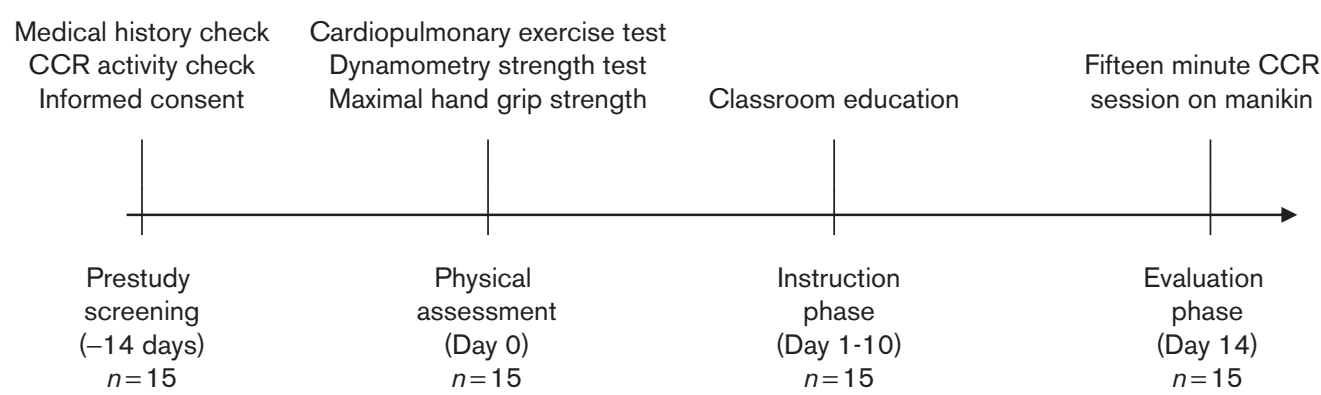

Study protocol. CCR, cardiocerebral resuscitation.

was calculated [13] by V-slope method, as executed in previous studies from our laboratory [14]. Ventilatory threshold is used to estimate the anaerobic threshold, which is the exercise intensity at which lactic acid starts to accumulate in the circulation (as a result of anaerobic metabolism). Maximal cycling power output was reported.

\section{Muscle strength}

In this test, maximal muscle strength (muscle's ability to generate one single peak force against a physical object) and muscle strength endurance (muscle's ability to continue to generate peak forces for a prolonged duration or during repeated contractions against a physical object) were assessed on an isokinetic dynamometer (Biodex Medical Systems Inc., New York, USA). Following a 5-min standardised warming-up (Technogym Lat Pull, Pectoral, ArmCurl), the participants were positioned and fixated in a semi supine $\left(15^{\circ}\right.$ backward inclination) sitting position (standardised shoulder and thorax fixation). To test maximal muscle strength each participant performed two maximal isometric elbow flexions and extensions (3 s) at an elbow angle of $90^{\circ}$ interspersed by 45 -s rest intervals. Maximal isometric torque at each angle was calculated as the average of the manually smoothed static torque curves. After a brief rest period muscle strength endurance was tested. The participants performed one bout of 20 maximal isokinetic $(180 \%$ s) elbow flexions and extensions from $90^{\circ}$ to $20^{\circ}$ (range of motion of $70^{\circ}$ ). After each contraction the arm was returned passively to the $90^{\circ}$ point. Muscle strength endurance was calculated as the percentage total work decrease from the first three contractions to the last three. All tests were performed unilaterally. Immediately after the muscle strength measurements, the same examiner asked the participants to perform dominant hand dynamometry (Jamar hand grip dynamometer; Sammons Preston Rolyan, Bolingbrook, Illinois, USA) two times [15]. The participants were standing upright with their elbows at $90^{\circ}$.

\section{Instruction phase}

During the instruction phase the participants received a standardised 10-min classroom-based CCR training course including theoretical education (short video: 'Red Cross Flanders') and skills practice. This course was provided by trained CCR instructors from the hospital.

\section{Evaluation phase}

Each participant was instructed to perform sustained chest-compression-only CGR up to $15 \mathrm{~min}$ or until exhaustion. No feedback about the CCR performance was provided to the participants. However, the participants were instructed that arms should be locked in extension. Resuscitation performance was assessed by Laerdal PC Skill Reporting System, connected to an adult CCR training manikin, providing compression compliance data, including numerical and graphical summaries. Compressions were recorded as correct if both depth and hand placements were in keeping with the standard 2005 guidelines [16], defining adequate compression depths to range within a $38-51 \mathrm{~mm}$ margin, adequate compression rate for adults to be at least 100 compressions/min. Hence, CCR quality is then defined as the number of compressions with adequate depth (between $38-51 \mathrm{~mm}$ ) within a certain timeframe (expressed as \%). Compression rates (external chest compression/min) and compression depths $(\mathrm{mm})$ were obtained after $1,3 \mathrm{~min}$, and for two consecutive time periods of $5 \mathrm{~min}$ (period 1: $5-10 \mathrm{~min}$, period 2: 10 $15 \mathrm{~min}$ ). The time frames for endpoint assessment are chosen according to the rapid defibrillation response interval: the time from start of basic life support by first responders to initiation of advanced life-support measures, including delivery of shocks by an automated external defibrillator [out of hospital (para)medic outreach team: $8-10 \mathrm{~min}$, public access automatic external defibrillator: $5 \mathrm{~min}$, in-hospital: 2-3 $\mathrm{min}$ ] [17-19].

During CCR heart rate was measured continuously by using a commercial heart rate monitor (Polar, Oy, Finland). Capillary blood samples were taken in duplicates at baseline and after 5,10 and 15 min to determine blood lactate levels $(\mathrm{mmol} / \mathrm{l})$ using an automated lactate analyser (Analox Instruments Ltd, London, UK). 


\section{Statistical analysis}

Data are expressed as means \pm standard deviation. First, changes of parameters (blood lactate level, heart rate, compression depth and frequency and CCR quality) during CCR were analysed by paired sample $t$-tests (by comparing with baseline measurement). Second, univariate relationships between variables (CCR quality with participants' characteristics) were examined with Spearman's rank correlation coefficients. Third, univariate relationships between variables (CCR quality with participants' characteristics) were further evaluated within the defined timeframes of CCR. Statistical significance was set at a $P$ value of less than 0.05 (two-sided). All calculations were performed using the Statistical Package for the Social Sciences, version 15.0 (IBM Corporation, New York, USA).

\section{Results}

Baseline CCR operator characteristics, exercise performance capacity and muscle strength are listed in Table 1. The participants were middle aged and had a normal BMI, good cardiopulmonary exercise capacity (based on $\%$ predicted $\mathrm{VO}_{2 \text { peak }}$ ) and general muscle strength (based on $\%$ predicted maximal hand grip strength).

In Figures 2a and 2b, changes in capillary blood lactate concentration and heart rate, respectively, during CCR are displayed. Blood lactate concentration increased with a trend ( $P$ between 0.05-0.06) ranging from 1.7 to $8.0 \mathrm{mmol} / \mathrm{l}$ following $15 \mathrm{~min}$ of $\mathrm{CCR}$, and heart rate increased significantly $(P<0.001)$ ranging from 45 to $93 \%$ of maximal heart rate. Baseline participant characteristics, cardiorespiratory exercise performance capacity, upper body dynamometry strength parameters and hand grip muscle strength, did not correlate significantly with blood lactate levels and heart rate during CCR $(P>0.05)$.

Averaged CCR-performance is shown in Fig. 3. Compression rate and CCR quality did not change from start up to $15 \mathrm{~min}(P>0.05)$. Compression depth lowered significantly from 1 up to $10 \mathrm{~min}$ of CCR $(P<0.05)$. The overall CCR quality, compression rate and compression depth was $36 \pm 34 \%, 117 \pm 21$ and $38 \pm 9 \mathrm{~mm}$, respectively. This corresponded to $577 \pm 306 \mathrm{~s}$ of inadequate chest compressions during $15 \mathrm{~min}$ of sustained CCR (900s). Between men and women, average compression depth ( $42 \pm 6$ vs. $29 \pm 6 \mathrm{~mm}$, respectively) was significantly different $(P<0.05)$. Compression rate was not different between the sexes $(115 \pm 24$ vs. $120 \pm 15$ compressions/min in men and women, respectively; $P>0.05)$.

Significant $(P<0.05)$ univariate correlations (see Table 1$)$ were found between overall $(15 \mathrm{~min})$ CCR quality and height $(r=0.53), \mathrm{VO}_{\text {2peak }}(r=0.54)$, maximal cycling power output $(r=0.54)$, ventilatory threshold $(r=0.67)$, isometric elbow extension peak torque at $90^{\circ}(r=0.55)$.
Fig. 2
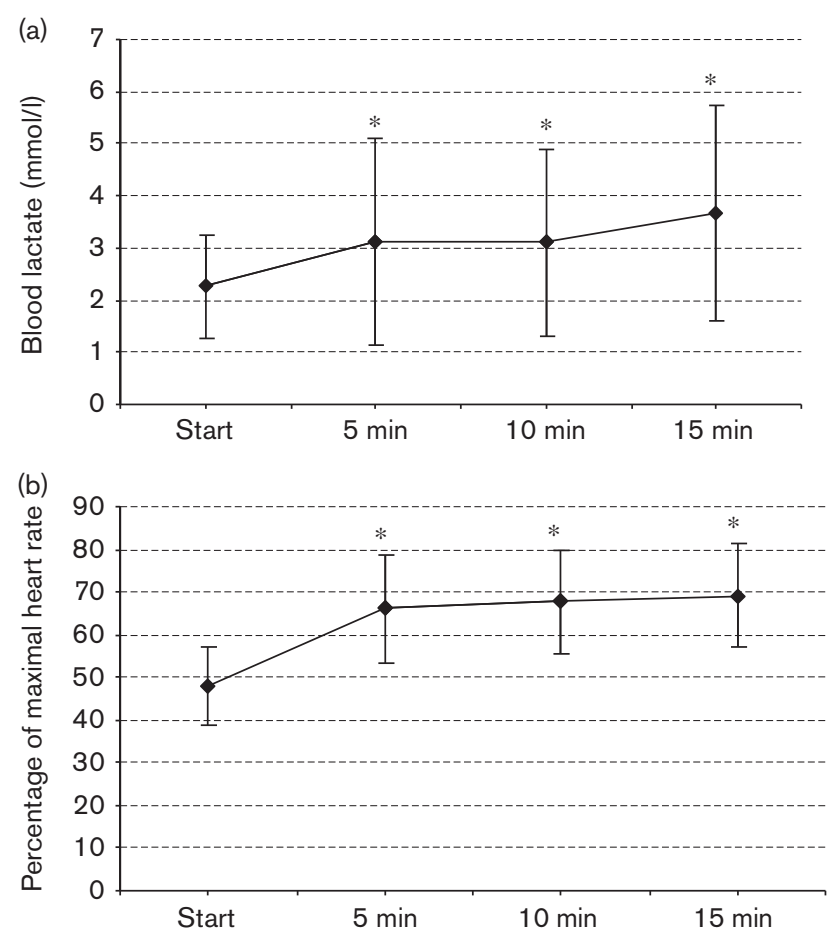

Physiological response to cardiocerebral resuscitation (CCR). (a) Change in capillary blood lactate concentration during CCR. *Different when compared with start ( $P$ between 0.05-0.06). (b) Change in heart rate during CCR. *Significantly different when compared with start $(P<0.05)$.

Between men and women, overall CCR quality ( $52 \pm 13$ vs. $5 \pm 5 \%$, respectively) was significantly different $(P<0.05)$. In Table 1 , correlation coefficients between CCR quality and the above-mentioned predictors are shown within different timeframes. The ventilatory threshold correlated significantly with CCR quality up to $5 \mathrm{~min}(P<0.05)$. After this timeframe, isometric elbow extension peak torque at $90^{\circ}$ correlated significantly with CCR quality $(P<0.05)$.

\section{Discussion}

From this study three findings emerged. First, cardiopulmonary (or physical) exercise capacity and muscle strength did not correlate significantly with blood lactate levels and heart rate during sustained CCR. Second, up to $5 \mathrm{~min}$ of sustained CCR, ventilatory threshold was associated with CCR quality. Third, after 5 min quality of CCR was related to maximal muscle strength.

Previous studies pointed at insufficient CCR performance in healthcare professionals and warranted exploration of factors related to such low performance $[5,20]$. It is proposed that rescuer physical fatigue might interfere with delivery of adequate chest compression rate and/or depth. Significant physical fatigue and shallow compressions are seen after already $1 \mathrm{~min}$ of CCR, although 

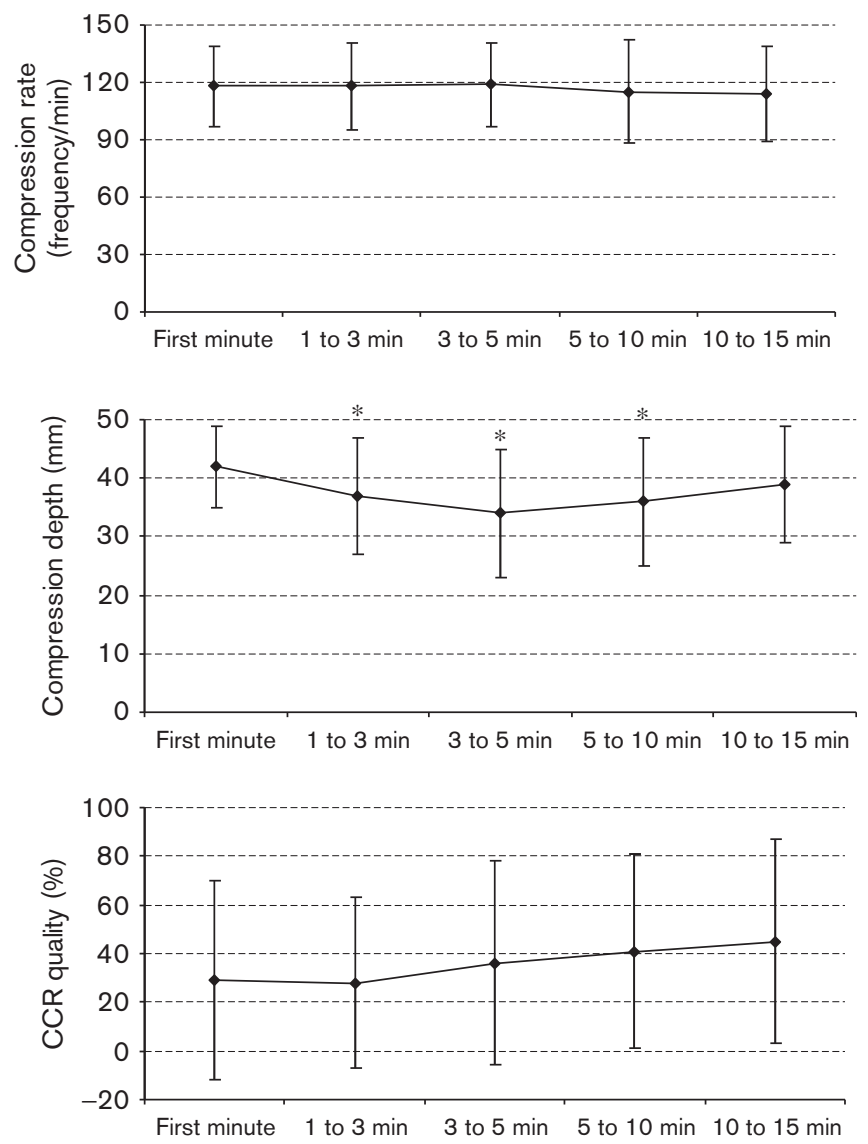

Cardiocerebral resuscitation performance. CCR, cardiocerebral resuscitation. *Significantly different when compared with start $(P<0.05)$.

rescuers may deny that physical fatigue is present for 5 min or more [5]. As a result, it might be speculated that a reduced cardiopulmonary exercise capacity and/or muscle strength interferes with proper CCR performance. In accordance, Lucia et al. [7] found that heart rate and oxygen uptake remained significantly lower in physically active healthcare professionals during sustained CCR, when compared with sedentary healthcare professionals. However, a different CCR quality was not demonstrated between the two groups. Their results indicate that a good physical fitness lowers the cardiopulmonary response to CCR, but it does not seem to affect CCR quality. As a result, it remained to be established whether there is a correlation between the components of physical fitness (cardiopulmonary exercise capacity and muscle strength) and CCR performance in healthcare professionals.

During sustained CCR blood lactate concentrations and heart rate increased significantly over time, and with comparable magnitude as reported in previous studies $[7,20,21]$. These markers of exercise intensity, as well as the appearance of shallow chest compressions, are repeatedly recorded concurrently after $1 \mathrm{~min}$ of sustained
CCR $[7,21,22]$. Noticeably, we were unable to predict changes in heart rate and blood lactate concentrations through participants' characteristics, cardiopulmonary exercise capacity and muscle strength. Even more importantly, blood lactate concentrations and heart rate were not related to CCR quality, which is the first important finding in this study. These data suggest that the cardiopulmonary and/or metabolic response during CCR does not reflect, or indicate, CCR quality as already described by Lucia et al. [7].

The ventilatory threshold was the only predictor for good CCR quality up to $5 \mathrm{~min}$, but not beyond this timeframe. Participants with a high-ventilatory threshold were more likely to achieve greater CCR quality. The ventilatory threshold is an estimation of the anaerobic threshold, and indicates the oxygen uptake level above which aerobic energy production is supplemented by anaerobic mechanism [13]. As a result, this threshold reflects the upper border of a working intensity, which can be sustained for a prolonged period. It follows that a high ventilatory threshold in healthcare professionals might increase the likelihood for greater CCR quality. This assumption requires future study in which the impact of 
an exercise intervention (to increase ventilatory threshold) on CCR quality will be assessed in healthcare professionals.

In our population, maximal upper extremity muscle strength predicted CCR quality after $5 \mathrm{~min}$. However, greater muscle strength did not predict good CCR quality in the first few min. According to guidelines, rescuers are advised to execute CCR in alternating cycles of $2 \mathrm{~min}$ [2]. As a result, the importance of maximal muscle strength on CCR quality in real-life situations seems limited. Our results indicate that in middle-aged adults, appropriate chest compression technique prevails to maximal muscle strength in performing high-quality CCR. This hypothesis needs further testing in a larger study population including youngsters and the elderly.

Taking the above-mentioned results together, it might be speculated that the ventilatory threshold rapidly loses its relation with CCR quality because the exercise level that is required for CCR is too high. In accordance, blood lactate concentrations rapidly rose to levels of $3.1 \pm 2.2 \mathrm{mmol} / \mathrm{l}$ within $5 \mathrm{~min}$ of CCR. Moreover, of the 15 participants, six showed a continued increase in blood lactate concentrations during $15 \mathrm{~min}$ of sustained CCR. As a result, CCR can only be sustained for a prolonged period by the addition of anaerobic capacity (which is reflected by greater maximal muscle strength). In accordance, the effect of maximal muscle strength on CCR quality is greater when CCR is prolonged. This hypothesis does, nonetheless, require further study.

Of major concern in our study was the fraction of inappropriate chest compressions within the first $5 \mathrm{~min}$ of CCR in well-trained hospital staff (overall CCR quality $36 \pm 34 \%$, corresponding to $577 \pm 306 \mathrm{~s}$ of inadequate chest compressions). Especially in our female participants, CCR quality was very low. In our protocol we did not provide 'in-time' feedback and monitoring of CCR quality during resuscitation efforts. These results mirror the percentages obtained in previous studies examining adequate chest compression fractions during CCR $[23,24]$. Consensus guidelines clearly define how CCR is to be performed [16,25]. Interruptions in CCR or failure to provide compressions during cardiac arrest ('noflow time') should be limited [26,27]. In animal studies, coronary and cerebral perfusion pressure, hemodynamic function and survival outcomes were adversely affected by even short pauses in chest compressions $[3,28]$. The impact of audiovisual feedback technology on the performance of CCR is a subject of further investigation.

The results from this study might have some clinical implications. On account of the relation between aerobic exercise capacity and CCR quality during the first few min, it might be important to stimulate/promote regular physical activity (mainly endurance-type exercise such as jogging, cycling, etc.) in healthcare professionals regularly involved in CCR. As the guidelines recommend alternating cycles of 2 min during CCR, a greater muscle strength (by means of strength training) might not contribute to greater GCR quality.

The relative small study sample may limit the robustness of some of our findings. However, our results and participants' characteristics are consistent with previous studies in this field. Patients with chronic disease were excluded from this study. These findings therefore do not apply to healthcare professionals with chronic disease.

In conclusion, during sustained CCR the ventilatory threshold is related to chest compression quality for up to $5 \mathrm{~min}$. After $5 \mathrm{~min}$ of sustained CCR, quality of chest compressions is related to maximal muscle strength.

\section{Acknowledgements}

The authors acknowledge the nurses and physicians who contributed to this research. This research was supported by a research Grant of the Jessa Hospital and Heart Centre Hasselt. Laerdal BeNeLux provided logistic support.

\section{References}

1 Wang HE, Min A, Hostler D, Chang CC, Callaway CW. Differential effects of out-of-hospital interventions on short and long-term survival after cardiopulmonary arrest. Resuscitation 2005; 67:69-74.

2 Ewy GA, Kern KB. Recent advances in cardiopulmonary resuscitation. J Am Coll Cardiol 2009; 53:149-157.

3 Wik L, Kramer-Johansen J, Myklebust H, Sørebø H, Svensson L, Fellows B, et al. Quality of cardiopulmonary resuscitation during out-of-hospital cardiac arrest. JAMA 2005; 293:299-304.

4 Abella BS, Alvarado JP, Myklebust H, Edelson DP, Barry A, O'Hearn N, et al. Quality of cardiopulmonary resuscitation during in-hospital cardiac arrest. JAMA 2005; 293:305-310.

5 Greingor JL. Quality of cardiac massage with ratio compression-ventilation 5/1 and 15/2. Resuscitation 2002; 55:263-267.

6 The American Heritage Dictionary of the English Language. 4th ed. Boston Houghton Mifflin Harcourt Publishing Company; 2010.

7 Lucia A, de las Heras JF, Perez M, Elvira JC, Carvajal A, Alvarez AJ, et al. The importance of physical fitness in the performance of adequate cardiopulmonary resuscitation. Chest 1999; 115:158-164.

8 Schlüssel MM, dos Anjos LA, de Vasconcellos MT, Kac G. Reference values for handgrip dynamometry for healthy individuals: a population-based study. Clin Nutr 2008; 27:601-607.

9 Wasserman K, Hansen JE, Sue DY, Casaburi R, Whipp BJ. Principles of exercise testing and interpretation. 3rd ed. Philadelphia: Lippincott Williams \& Wilkins; 1999.

10 Buchfuhrer MJ, Hansen JE, Robinson TE, Sue DY, Wasserman K, Whipp BJ. Optimizing the exercise protocol for cardiopulmonary assessment. $J$ Appl Physiol 1983; 55:1558-1564.

11 Hansen D, Dendale P, Berger J, Onkelinx S, Reyckers I, Hermans A, et al. Importance of exercise training session duration in the rehabilitation of coronary artery disease patients. Eur J Cardiovasc Prev Rehabil 2008; 15:453-459.

12 Fletcher GF, Balady GJ, Amsterdam EA, Chaitman B, Eckel R, Fleg J, et al. Exercise standards for testing and training: a statement for healthcare professionals from the American Heart Association. Circulation 2001; 104:1694-1740.

13 Beaver WL, Wasserman K, Whipp BJ. A new method for detecting anaerobic threshold by gas exchange. J App/ Physiol 1986; 60:2020-2027.

14 Hansen D, Dendale P, Berger J, Meeusen R. Low agreement of ventilatory threshold between training modes in cardiac patients. Eur J Appl Physiol 2007; $101: 547-554$.

15 Bohannon RW, Peolsson A, Massey-Westropp N, Desrosiers J, BearLehman J. Reference values for adult grip strength measured with a Jamar dynamometer: a descriptive meta-analysis. Physiotherapy 2006; 92:11-15. 
16 ECC Committee, ECC Subcommittee, ECC Task Force. International consensus on cardiopulmonary resuscitation (CPR) and emergency cardiovascular care (ECC) science with treatment recommendations. Circulation 2005; 112 (Suppl):IV19-IV34.

17 Stiell IG, Wells GA, Field B, Spaite DW, Nesbitt LP, De Maio VJ, et al. Advanced cardiac life support in out-of-hospital cardiac arrest. N Engl J Med 2004; 351:647-656.

18 Valenzuela TD, Roe DJ, Nichol G, Clark LL, Spaite DW, Hardman RG. Outcomes of rapid defibrillation by security officers after cardiac arrest in casinos. N Engl J Med 2000; 343:1206-1209.

19 Caffrey SL, Willoughby PJ, Pepe PE, Becker LB. Public use of automated external defibrillators. N Engl J Med 2002; 347:1242-1247.

20 Riera SQ, Gonzalez BS, Alvarez JT, Fernández Mdel M, Saura JM. The physiological effect on rescuers of doing 2 min of uninterrupted chest compressions. Resuscitation 2007; 74:108-112.

21 Lonergan JH, Youngberg JZ, Kaplan JA. Cardiopulmonary resuscitation: physical stress on the rescuer. Crit Care Med 1981; 9:793-795.

22 Bridgewater FH, Zeitz C, Field J, Inglis A, Poulish K. The impact of the ILCOR 2005 CPR guidelines on a physical fitness assessment: a comparison of old and new protocols. Resuscitation 2008;

76:405-412.
23 Ashton A, McCluskey A, Gwinnutt CL, Keenan AM. Effect of rescuer fatigue on performance of continuous external chest compressions over $3 \mathrm{~min}$. Resuscitation 2002; 55:151-155.

24 Aufderheide TP, Pirrallo RG, Yannopoulos D, Klein JP, von Briesen C, Sparks CW, et al. Incomplete chest wall decompression: a clinical evaluation of CPR performance by trained laypersons and an assessment of alternative manual chest compression-decompression techniques. Resuscitation 2006; 71:341-351.

25 Founding Members of the International Liaison Committee on Resuscitation. The International Liaison Committee on Resuscitation (ILCOR) - past and present. Resuscitation 2005; 67:157-161.

26 Berg RA, Sanders AB, Kern KB, Hilwig RW, Heidenreich JW, Porter ME, et al. Adverse hemodynamic effects of interrupting chest compressions for rescue breathing during cardiopulmonary resuscitation for ventricular fibrillation cardiac arrest. Circulation 2001; 104:2465-2470.

27 Christenson J, Andrusiek D, Everson-Stewart S, Kudenchuk P, Hostler D, Powell J, et al. Chest compression fraction determines survival in patients with out-of-hospital ventricular fibrillation. Circulation 2009; 120:1241-1247.

28 Sato Y, Weil MH, Sun S, Tang W, Xie J, Noc M, et al. Adverse effects of interrupting precordial compression during cardiopulmonary resuscitation. Crit Care Med 1997; 25:733-736. 